1,718,132 person-years at risk (mean follow-up 12.8 years), 667 individuals had a hospital record of PAD (3.88 per 10,000 person-years). Job strain was associated with a 1.41fold increase (95\% confidence interval: 1.11 to 1.80 ) in the average risk of hospitalisation with $\mathrm{PAD}$, independently of age, sex, socioeconomic position and lifestyle-related characteristics. The study-specific estimates were moderately heterogeneous ( $\tau$-squared $=0.0427$, I-squared: $26.9 \%)$, but we found no clear evidence of sex, socioeconomic position, smoking or baseline diabetes explaining the heterogeneity. The average absolute risk difference between the job strain and no strain groups was firmly positive $(3.28$, 95\% confidence interval: 0.78 to 5.78 ).

Discussion Job strain, a marker of psychosocial stress at work, was associated with small but consistent increase in the risk of hospitalisation with PAD. The risk estimates for PAD were similar in magnitude to those previously estimated for coronary heart disease and ischaemic stroke, suggesting that job strain is at least as important a risk factor for PAD as it is for these severe cardiovascular disease outcomes. Our findings highlight the need for physicians in occupational health and primary care to recognise and target work-related stress as a risk factor for PAD.

\section{P61 PREVALENCE OF DEPRESSION AND ITS ASSOCIATION WITH VIGOROUS PHYSICAL ACTIVITY (VPA) IN THE ENGLISH POLICE FORCE: A QUANTITATIVE SECONDARY DATA ANALYSIS OF THE AIRWAVE DATASET}

R Maqsood*. The European Centre for Environment and Human Health, University of Exeter, Truro, UK

\subsection{6/jech-2020-SSMabstracts. 154}

Background Given the increasingly sedentary nature of policing and the subsequent rise in depression rates among police officers, physical inactivity is considered as a risk factor for depression. In contrast, Vigorous Physical Activity (VPA) is preventative against depression, yet this has been limitedly studied in policing. This study primarily aimed to explore the prevalence of depression and examine its association with VPA in the English police force, further stratified by gender. The secondary aims included examining the impact of sociodemographic and lifestyle variables on the prevalence of depression.

Methods A cross-sectional, secondary data-analysis of the Airwave (the largest cohort-dataset for the UK's police forces) was undertaken using Stata. The descriptive statistics were used to explore the socio-demographic profile of the sample $(n=28,465)$. The multivariate logistic regression models were run to examine the association between depression and VPA, reporting Odds Ratio (OR) with 95\% Confidence Interval (CI) at significance level of 5\%, while controlling the covariates.

Results The prevalence of depression was 12\% in the English police force and $25 \%$ of police employees were physically inactive. The multivariate logistic regression analysis revealed that relative to physically inactive participants, those who did VPA for either 4 or 7 days had equally the lowest odds of reporting depression (OR 0.59, 95\% CI: 0.51-0.70, p<0.001) (OR $0.59,95 \%$ CI: $0.45-0.77, \mathrm{p}<0.001$ ) respectively, suggesting a U-shaped relationship; which was also observed in the gender-stratification analysis. While the odds of reporting depression were significantly lower for men than women, VPA was significantly associated with reducing the odds of reporting depression slightly more in women (OR 0.43, 95\% CI: $0.27-0.67, \mathrm{p}<0.001)$ than in men (OR 0.50, 95\% CI: $0.33-$ $0.75, \mathrm{p}<0.01)$, indicating women may be more likely to benefit from VPA than men.

Conclusion VPA may significantly reduce the odds of reporting doctor-diagnosed depression in a U-shaped trend. Being physically active for 4 days may be as beneficial against depression as being active for 7 days. VPA may be more protective against depression for policewomen than policemen. Limited by cross-sectional design, the causal pathway between depression and VPA could not be established. Future research warrants longitudinal study design using objectively measured physical activity and clinically measured depression to fully understand their dose-response relationship. The policymakers may use these findings to design physical-activity guidelines for the English police force. The leadership of the English police force is also recommended to augment police officers' recruitment and annual fitness tests with mental-health assessments.

\section{P62 THE IMPACT OF AREA DEPRIVATION ON ENGAGEMENT IN ARTS AND CULTURAL ACTIVITIES: EVIDENCE FROM A UK NATIONALLY-REPRESENTATIVE STUDY}

${ }^{1} \mathrm{HW}$ Mak*, ${ }^{2} \mathrm{R}$ Coulter, ${ }^{1} \mathrm{D}$ Fancourt. 'Department of Behavioural Science and Health, University College London, London, UK; ${ }^{2}$ Department of Geography, University College London, London, UK

\subsection{6/jech-2020-SSMabstracts. 155}

Background There is a growing body of literature suggesting that arts and cultural engagement can benefit physical, mental and social wellbeing. However, there is evidence that there is a social and geographical gradient across the engagement; more likely to be engaged by people with higher educational qualifications and those living in affluent countryside and cosmopolitan neighbourhoods. But it remains unclear precisely why this might be. Examining the geographical patterns of arts engagement has particular policy relevance at present, given the growing interest in the 'social prescribing' scheme, a place-based health approach, and in place-based funding in arts in health research and programmes. However, as yet, there has been little research into whether place has an effect on patterns of arts and cultural engagement independent of individual factors. Therefore, in this study, we sought to disentangle associations between geographical deprivation and arts engagement from individual measures of socio-economic positions by using the propensity score matching (PSM) technique.

Methods We analysed data from 18,587 participants in the Understanding Society Wave 2 and used PSM to match individuals who lived in the $30 \%$ most deprived areas with those who lived in the $30 \%$ least deprived areas and shared similar demographic background and socio-economic characteristics. The level of area deprivation was derived from the Index of Multiple Deprivation (IMD), which measures the relative deprivation for small areas based on seven domains (e.g. crime and employment). Three different types of arts and cultural engagement were explored: arts activities (e.g. dancing), cultural events attendance (e.g. exhibition), and museums and heritages visits. 